For the period Nov. 1, 1987 - Jan. 31, 198/8

Work Performed under Contract No. DE-AC22-84PC70768

For

U. S. Department of Energy

Pittsburgh Energy Technology Center

P. O. Box 10940

Pittsburgh, PA 15236

By

United Technologies Research Center

East Hartford, CT 06108

\title{
DISCLAIMER
}

This report was prepared as an account of work sponsored by an agency of the United States Government. Neither the United States Government nor any agency thereof, nor any of their employees, makes any warranty, express or implied, or assumes any legal liability or responsibility for the accuracy, completeness, or usefulness of any information, apparatus, product, or process disclosed, or represents that its use would not infringe privately owned rights. Reference here:.: to any specific commercial product, process, or service by trade name, trademark, manufacturer, or otherwise does not necessarily constitute or imply its endorsement, recommendation, or favoring by the United States Government or any agency thereof. The views and opinions of authors expressed herein do not necessarily state or reflect those of the United States Government or any agency thereof. 
Report Distribution List

DOE Contract No. DE-AC22-84PC70768

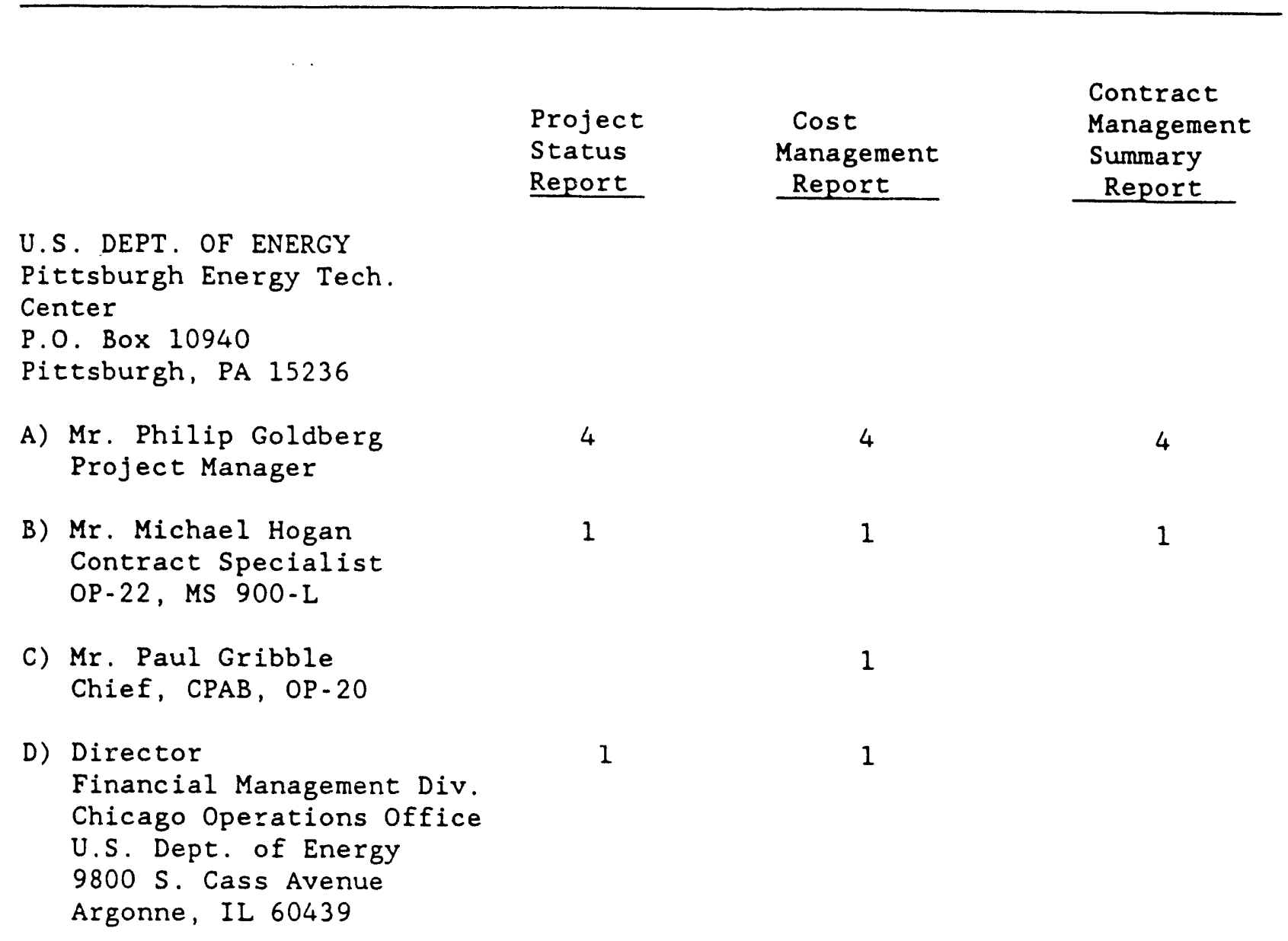




\section{KINETICS OF COAL DEVOLATILIZATION}

Inspection of tar evolution data from heated grid investigations indicates a 500 degree discrepancy in reported temperatures of tar evolution from HV bituminous coal samples. For reportedly equivalent heating conditions - $1000 \mathrm{c} / \mathrm{sec}$ heating to peak temperatures in one atmosphere of helium, zero hold time - - some investigators report half the tar evolved at temperatures of $500 \mathrm{~K}$ while others report peak temperatures of nearly $1100 \mathrm{~K}$ are necessary to achieve the same extent of tar evolution.

Despite significant differences in the extent of tar evolution with temperature in these investigations, the reported tar evolution data have several characteristics in common: sigmoid evolution curves with respect to peak temperature; similar temperature differentials of tar evolution and, therefore, similar times of tar evolution during the transient heating process; approximately the same asymptotic tar yields for similar coal types. These similarities in tar evolution yield characteristics in transient heating conditions imply: a.) the coal particles experience nearly the same heat flux rates in the tar evolution stage; and $b$. ) the differences in absolute temperatures of tar evolution for similar coal types are due to the difficulties in measuring particle temperatures during such transient heating experiments. In all cases, the measured temperature is a thermocouple "placed near" or "with" the coal sample. It is assumed the coal particles follow the same transient temperature trajectory.

The results indicate that the thermocouple heating rate $(1000 \mathrm{c} / \mathrm{sec})$, that is, the grid to sample heat flux rate, is the common element in the experiments. The actual heating rate of the sample, relative to the system thermocouple, depends on the sample-to-grid surface area load factor and the intrinsic properties of the sample relative to the thermocouple. Calibration runs performed with nonvolatile and volatile "reference" material in the UTRC heated grid under similar heating conditions indicates the sample load factor and thermophysical properties are critical in determining the actual sample temperature relative to the thermocouple. Thus, it is not surprising that tar evolution times from the different investigations are similar, but absolute temperatures of tar evolution are substantially different in transient heating, zero-hold time experiments. The temperature dependence of tar yields for long hold time conditions in the different investigations are in much closer agreement, indicating that given enough time the thermocouple and sample reach thermal equilibrium, as expected, in the various systems.

UTRC results for a PSCC1451D, 63-75 micron particles, indicate tar evolution occuring in zero-hold time heating conditions over peak thermocouple temperatures of $450 \mathrm{C}$ to $625 \mathrm{C}$. Long hold time experiments indicate the tar evolution occurs from $350 \mathrm{C}$ to $600 \mathrm{C}$, with both heating conditions displaying nearly similar yields if $600 \mathrm{C}$ peak thermocouple temperatures are achieved, irrespective of holding time. Given the 50 - 100 degree uncertainty in sample temperature relative to thermocouple during transient heating, it appears the higher temperatures of tar evolution during the zero hold time experiments are in large part due to differences in sample and thermocouple temperature trajectories during the tar evolution period in transient heating experiments. 
Such differences in sample and thermocouple temperatures during rapid, transient heating conditions are not unexpected in view of the fact that UTRC heat transfer investigations of heated grids indicate power dissipation from grid surface to be of the order of 0.5 - 5 watts $/ \mathrm{sq} . \mathrm{cm}$. in helium gas over reported temperatures of tar evolution. In transient heating conditions, sample particle and thermocouple bead temperatures can only be expected to be identical if the sample "loads" the grid in a manner identical to a

thermocouple. That is, the transient temperature response of the coal particles to this heat flux rate will be the same as the thermocouple if the sample, places the same thermal inertia on the grid "surface"; has the same heat transfer properties as the thermocouple bead relative to the grid mesh; tar evolution is thermally neutral; the tar evolution does not interfere with the heat transfer mechanism between the grid and the coal particles. The similarity in tar evolution characteristics indicated by various investigators using heated grids - shape of tar evolution curve, time of tar evolution given a grid heating rate, asymptotic tar yields, temperature sensitivity of long hoid time tar yields - in the context of significant differences in tar evolution temperatures in transient heating experiments can be interpreted by a detailed understanding of the heat transfer conditions within such experiments. 
Task 2.1 Engineering Kinetics of Coal Devolatilization (Ohio State)

No report submitted to UTRC.

Task 2.2 Fundamental Mechanisms and Kinetics of Volatiles Formation and Evolution

Tar Evolution in Heated Grid Apparatuses

The wire mesh technique has been employed for over twenty years in investigating the devolatilization of pulverized coal. The technique is useful in that it provides quantitative data on the product distributions produced in semi-disperse, rapid heating of small coal particles. However, as commonly observed for a range of devolatilization experiments using different heating techniques, the temperature sensitivity of tar yield results are not in apparent agreement. The transient, heterogenous nature of the process and the chemical complexity of the parent material and heavy hydrocarbon volatiles establish experimental difficulties and prevent immediate interpretation of observable behavior. Heavy hydrocarbons, tars, are the major volatile species evolved during the transient particle heating to temperatures in excess of 600 $C$. It is the formation and evolution of these species that are the natural references in attempts to discover the causes for the reported differences.

More specifically, it is the evolution of tars during experiments characterized by nonisothermal temperature profiles that result in the greatest differences in reported results. Figure 1 displays tar yields as a function of peak temperatures observed for some high volatile bituminous coals. All of these experiments were performed using "zero hold time" conditions, that is, the system thermocouple was heated to the given temperature and the electrical power immediately terminated. In each of the experiments in Figure 1, thermocouple heating rates of $1000 \mathrm{C} / \mathrm{sec}$ were obtained in one atmosphere of helium gas. In only one case (1), was a carrier flow maintained over the mesh-sample surface. In some cases, natural convection and radiation cooling are the main modes of sample cooling $(2,3,4$,$) . In other cases an auxiliary quench was employed to increase the$ mesh-sample cooling rate (1,5, this study). Natural convection and radiation provides cooling rates of the order of several hundred degrees per second, whereas forced convection cooling produces cooling rates in excess of 1000 $\mathrm{C} / \mathrm{sec}$. However, a recent study has indicated total yields, and, by implication, tar yields are insensitive for cooling rates greater than 50 $\mathrm{c} / \sec (6)$.

Table I lists the reported elemental compositions of the coals employed and Figure 2 shows their location on a coalification band plot relative to the "reference" coal of this investigation, PSOC 1451D. Also shown are the location of the other bituminous coals investigated in this program. Inspection of Figure 1 indicates asymptotic tar yields of the Pittsburgh Seam high volatile bituminous coal vary from 238 to approximately 328 . The 378 tar yield observed for the UK coal consists of "heavy" tars and "light" tars, with heavy tars comprising approximately 708 of this amount. With this distinction in yield definition, the asymptotic, easily condensible, "heavy," tar yields vary by only 338 from one investigation to the next. 
From a combustion modeling perspective, the more important isssue is the apparent discrepancy in the temperature dependence of the tar yield in transient heating conditions. Reported temperatures for half the potential tar evolution range from as low as $575 \mathrm{~K}$ to as high as $1125 \mathrm{~K}$. Despite the pronounced variation in reported temperatures of tar evolution, the tar evolution curves have several characteristics in common: they are sigmoid in appearance, that is, the temperature sensitivity of the tar evolution varies with extent of evolution; the reported temperature sensitivities of the tar yield, that is, the change in tar yield per unit change in peak temperature, do not vary as widely as the absolute temperatures; the major fraction of the tar yield occurs in an approximately 250 degree temperature range; asymptotic tar yields do not vary appreciably with investigation.

An alternative manner of visualizing the agreement in the data despite the obvious, absolute temperature differences is illustrated in Figure 3 . In this figure the empirical tar evolution times are plotted versus the reported temperatures for the midpoint of tar evolution. As implied in the discussion above, the investigators are in substantial agreement in the tar evolution times, given the common heating conditions, but disagree concerning the temperature of tar evolution. The essential agreement in tar evolution times, temperature sensitivity of tar evolution, and asymptotic tar yields imply the coal samples were experiencing similar heat flux rates during the main phase of tar evolution. Such results also imply that the measured temperature is a.) not always representative of the sample temperature during the transient tar evolution process; b.) only one of several important experimental parameters in determining the rate of tar evolution in such an experiment; c.) some combination of these two.

\section{Tar Evolution Times in Different Reactor Types}

If one widens the consideration of tar evolution phenomena to other heating techniques, the temperature discrepancies become even more apparent. The scatter in the plots of Arrhenius rate constants for various devolatilization apparatuses is well known. Some scatter arises from the differences in the model premises employed and the particular aspect of devolatilization considered. However, as in the heated grid results, but to a wider extent, a great deal of the scatter undoubtedly arises from differences in the assigned temperatures of tar evolution. The differences in the Arrhenius type correlations of the devolatilization rates obscures the rather signficant agreement in the tar evolution times if one considers in detail the conditions of heating involved in each experiment. More specifically, if one considers the rate of heat transfer to the devolatilizing coal particles during the tar evolution phase of coal pyrolysis, one finds the tar evolution times correlate strongly with the heat transfer rate -. Figure 4 -. assuming the bulk of tar release occurs during the transient particle heating from 300 to $600 \mathrm{C}$.

For experiments in whicki an associated thermocouple is taken to be a monitor of the reactor-to-sample heat flux rate, tar evolution is directly observed to occur from estimated particle temperatures of 300 to $600 \mathrm{C}$.

However, in rapid heating rate experiments -. 10,000 C/sec and above, net 
particle surface flux rates of 30 watts $/ \mathrm{sq} . \mathrm{cm}$. and above - tar evolution during the transient heating through $600 \mathrm{C}$ is implied by the data, but not directly observed due to experimental difficulties in measuring transient particle temperatures and differential tar yields.

Consequently, as observed in results for heated grid experiments alone, comparable heating conditions should produce comparable tar evolution times, but significantly different heating conditions should produce correspondingly different tar evolution times, provided the asymptotic particle temperatures are greater than approximately $600 \mathrm{C}$. The empirical correlation of Figure 4 directly indicates this is the case for subbituminous and bituminous coals, coals having significant tar yields in the early stages of pyrolysis. That is, transient tar evolution times are directly related to net flux rates delivered to the particle surface. The correlation does not explain the rather significant differences in reported temperature differences in tar evolution, observed for any particular experimental configuration or among the different experimental configurations employed.

\section{UTRC Heated Grid Data: Zero-Hold-Time Conditions}

Figure 5 displays the zero-hold-time tar evolution observed for $63-75$ micron particles of PSOC 1451D in one atmosphere of helium and with auxiliary quench following obtainment of the peak temperature. This UTRC data agrees most closely with the results of Unger and Suuberg (3) and Oh (4). It does not agree with the results of Desypris, et. al.(1), an earlier study performed by Suuberg (2), or a more recent study performed by Bautista, et. al. (5). Although zero-hold-time tar yields as a function of peak temperatures are not given by Gibbins-Matham as a function of peak temperature, the zero hold time total weight loss is reported as approximately 328 , with 30 sec hold time values only increasing to approximately 448. The tar yield at $30 \mathrm{sec}$ in 1 atmosphere of helium is given as 328 . In other words, the data of reference 6 strongly supports the Unger, Oh, UTRC results which indicate the major fraction of tar release from bituminous coals occurs between 400 and $600 \mathrm{C}$ in these heating conditions. The tar evolution temperatures of Reference 1 appear approximately 300 degrees lower, while those of References 2 and 5 appear 300 degrees higher. Since tar yields comprise the major fraction of the volatiles release from high volatile bituminous coals in these heating conditions, similar discrepancies are maintained with respect to total weight loss as a function of peak temperature. A comprehensive analysis of the literature with respect to the temperature dependence of weight loss in heated grid apparatuses in an independent laboratory has indicated this is the case (7).

Given the similarity in coal types and heating conditions, the substantial differences in tar evolution temperatures for zero-hold-time temperature profiles are undoubtedly more apparent than actual. "Calibration" of the UTRC heated grid device using samples of known specific heat, melting points and heats of fusion has indicated the importance of sample loading in determining accurate sample temperature measurements from associated thermocouple measurements. More specifically, it is the sample loading per unit superficial surface area of the wire mesh, relative to the thermocouple 
position, that determines the transient sample temperature relative to the measured thermocouple temperature. It is observed that, depending on the specific heat and latent heat of fusion of the reference material, sample distribution, relative to the thermocouple location, can signficantly affect the measured phase change temperatures.

Lower phase change temperatures are obtained by locating the thermocouple in the center of a locally dense load of sample. Higher temperatures are obtained by a thermocouple bead located on the "edge" of a sample covered area or at a position somewhat removed from the sample distribution. In the former case the sample overloads the grid locally, given the limited heat generation capability of the wire mesh. The overload causes the sample to melt nonuniformly from an area of high heat density at the sample edge inward toward the sample center. The transient temperature inflection in the thermocouple occurs at a temperature lower than the actual melting temperature of the material. In the latter case, the thermocouple measures the temperature of a basically "underloaded" screen area, resulting in a thermocouple inflection at a temperature greater than the actual melting temperature or, in the extreme case, simply missing any evidence of physcial change.

The local nature of the sample load effect is demonstrated by placing a thermocouple at a representatively loaded area of the screen and several millimeters away from a sample loaded area. In this case the "displaced" thermocouple records basically a blank screen transient temperature profile, whereas the sample-loaded thermocouple records a temperature nearer that local sample temperature, depending on the relative thermophysical properties of the screen and sample.

In transient coal devolatilization experiments, the particles load the grid in nonuniform packets, resulting in nonuniform, local load factors across the grid within a given run. In addition high heating rate experiments cause mechanical displacement of the grid surface changing the local load factors during a run. Plasticizing coals signficantly change particle size and shape within the grid as they change mass, further complicating the local load pattern for a given sample. Thus it is not surprising that signficant temperature differences are noted in the zero-hold-time data of the various investigators and that there is significantly less divergence in the long hold time tar evolution data, that is, in experiments wherein thermal equilibrium is established across the screen and, therefore, between the sample, grid material and thermocouple.

\section{UTRC Heated Grid Data: Varying Hold Time Data}

Tar yields for $100 \mathrm{sec}$ hold times at peak temperatures achieved by 1000 $\mathrm{C} / \mathrm{sec}$ heating in helium are given in Figure 6 for the PSOC $1451 \mathrm{D}$ coal in the UTRC heated grid reactor. Also shown are data for shorter hold times, and low pressure, long hold time tar yields together with the transient, zero-hold-time data for the same coal. The $100 \mathrm{sec}$ hold time results indicate ambient pressure affects the high temperature, $600+C$, asymptotic tar yield to a greater extent than the lower temperature, but temperature sensitive tar 
yields. The "ultimate" low pressure tar yield appears to be about 358 of the iarent coal mass compared to 308 for one atmosphere of helium. At hold temperatures below $450 \mathrm{C}$ it is difficult to distinguish between "vaccum" and atmospheric pressure tar yields. In other words, the higher temperature, heavier molecular. weight tar yields appear to be influenced more by ambient pressure conditions than the low temperature, lighter tar species.

Distinctions in vacuum and pressure produced tar yields become more pronounced at asymptotic particle temperatures of $500 \mathrm{C}$ or above. There is an interphase mass transfer resistance to the evolution of high temperature, heary molecular weight tars.

With respect to thermocouple hold time for a given thermocouple temperature, there appears to be some differences in tar yields observed for $50 \mathrm{sec}$ hold times versus $100 \mathrm{sec}$ hold times, with the longer hold times producing slightly greater yields. Whether the reduction in tar yield with decrease in hold times long relative to the zero-hold-time conditions is due to intraparticle chemical kinetic formation rates, interphase mass transfer resistance, or some combination of factors is not immediately apparent.

Intermediate temper:ture, $300 \mathrm{C}$ to $600 \mathrm{C}$, tar yields are ubviously most sensitive to transient heating conditions. Zero hold time tar yields are a small fraction of the yields observed for 50 and $100 \mathrm{sec}$ hold times at thermocouple temperatures in this temperature span. At $500 \mathrm{C}$ peak thermocouple temperature the zero hold time tar yield is only about 0.2 that of the corresponding long hold time yield. Differences between yields decrease with peak thermocouple temperature, the zero hold time yield at $600 \mathrm{C}$ being about 0.8 that of the corresponding long hold time yield. As noted above, high temperature, zero-hold-time yields can not be made to match the long hold time yields by simply an increase in peak thermocouple temperature. Yields converge to within 208 of each other at $600 \mathrm{C}$. For peak temperatures greater than $700 \mathrm{C}$, it appears there may be a decrease in tar yield in these heating rate conditions. Such a decrease would be associated with high temperature pyrolysis of heavy tars which are prevented from particle desorption by mass transfer resistances.

The differences in thermocouple temperature sensitivity of tar evolution for zero versus long hold times for thermocouple temperatures less than $600 \mathrm{C}$ is attributed in part to same factors causing the apparent differences in temperature sensitivity among different laboratories. If average peak sample temperatures during the zero-hold-time experiments were 75 - 100 degrees lower than the peak thermocouple, wire mesh, temperatures, the zero-hold-time tar evolution yields would be everywhere nearly within 258 of the long hold time yields.

\section{Discussion of Results}

As Figure 4 indicates, tar evolution times do vary signficantly with changes in heating conditions, which vary signficantly with reactor type or with programmed heating rate within a particular reactor. The range of heat transfer rates to coal particles during devolatilization in different reactor types and for the particle temperatures of major tar evolution are indicated 
in Figure 7. There is no direct evidence indicating the major fraction of tar evolution from high volatile bituminous coals can be shifted to significantly different particle temperatures by a change in 1.) thermocouple heating rate in nonisothermal experiments; or 2.) reactor temperatures in entrained flow systems. But there is a growing body of evidence indicating the integrated times of tar evolution, the instantaneous rates of tar release, and the molecular nature of tars vary directly with the heat transfer rate during the particle temperature rise through $600 \mathrm{C}$ to $700 \mathrm{C}$ and as implied by Figure 4 . The substantial differences in reported tar evolution temperatures for similar coal types, either for a given reactor type or for differenct reactor types, is probably due to incorrectly estimating transient particle temperatures from transient thermocouple temperatures or fixed reactor system temperatures. Since tar loss is such a major fraction of mass loss for bituminous coals weight loss kinetic models would immediately reflect the variations in reported particle temperatures.

With respect to transient particle mass loss via tar evolution, it appears parameters other than chemical kinetic have a substantial influence on the observed mass loss rates. Chemical kinetic considerations undoubtedly determine the molecular nature of the evolved tars, given a specific set of heat and mass transport related conditions. But with respect to particle mass loss, it appears heat transport related factors play a dominating role in determining the evolution rates of particle mass as some type of tar.

Heat flux rates determine the rate of particle mass loss as tar. The specific heating condtions - net heat flux rate, mode of heating, asymptotic particle temperature, ambient pressure - the type of tars evolved. From this perspective Figure 4 is misleading in the same manner that "universal", chemical kinetic models of coal devolatilization are misleading. That is, it implies that the chemical nature of the evolved tars and their mode of desorption are invariant with changes in heating conditions, that some invariant set of chemical pathways produce an invariant set of tars that evolve during coal devolatilization.

The intra-particle chemical kinetics needed to produce tar species capable of desorbing, given a net heat flux rate to the particle surface, is not the rate-limiting phenomena for particle mass loss via tar evolution for peak particle temperatures to approximately $600 \mathrm{C}$. If it were, there should be substantial offsecs in the temperature of tar evolution with heating conditions while continuing to produce the tars of the same chemical characteristics. This is not observed. The molecular nature of the evol.ved heavy hydrocarbons apparently varies with heating rate, indicating a multiplicity of physical and chemical detachment mechanisms are available within the coal mass to form tars capable of desorbing. At such solid or liquid phase temperatures over such a range, it is probable that a number of chemical and physical pathways are available for the formation of large organic molecules capable of desorbing.

The heating rate determines a.) the particular chemical and physical detachment pathway of intraparticle tar precursor formation; and b.) the allowable mass range of tars that can escape the particle by increasing the number of modes of desorption. The greater the apparent heating rate of the 
particle, net heat flux rate to the particle surface, the more desorption modes available to the range of detached tar species in the particle. Very slow heating appears to be dominated by vaporization phenomena. Intermediate heating rates appear to produce convective "despurption" of tars with gases through a plastic.melt in addition to vaporization. High surface power densities created by flash lamps and lasers appear capable of producing a non-equilibrium desorption of heavy molecular weight species as is observed in laser desorption of large, thermally labile organic species from substrate surfaces $(7,8,9)$. In short the higher the apparent heating rate the more tar desorption modes available for species too heavy to desorb in slow heating rate conditions.

In the heat flux rate conditions created by heated grid apparatuses, particle mass loss by tar evolution appears dominated by heating rate through particle temperatures of $600 \mathrm{C}$. Tar evolution rates at temperatures greater than $600 \mathrm{C}$ appears dominated by mass transfer factors. Rapid heating through higher particle temperatures causes intraparticle cracking of heavy molecular weight tars prevented from particle escape due to mass transfer resistance. Correlation of tar evolution times from a wide range of reactors with heat transfer rates substantially supports a sequential phase model of tar evolution - Figures 8 and 9 . In this sequential view of tar evolution, particle mass loss via tar release is dominated by heat transfer considerations through particle temperatures of approximately $600 \mathrm{C}$ (phases I and II of Figures 8 and 9 ) and mass transfer considerations for particle temperatures greater than $600 \mathrm{C}$ (phase III). Chemical kinetic considerations dominated the relativley slow degassing of the virgin char (phase IV of particle mass loss). It is important to keep in mind that although transport factors dominate the rate of mass loss via tar evolution, chemical kinetic mechanisms obviously determine the nature of the tars produced, given a particular set of heating conditions. Equally important, however, is the realization that a simple, universal chemical kinetic model of coal devoaltilization is meaningless over a wide range of heating conditions because tar evolution rates and tar properties are so intimately coupled to heating conditions, that is, transport factors.

Substantial differences in zero-hold-time versues long hold time data within a particular apparatus appears due to differences in thermocouple versus actual particle temperature due to the manrer in which the sample locally "loads" the wire mesh relative to the thermocouple. Differences in the temperature sensitivity of tar evolution for high volatile bituminous coals among different investigators for zero-hold-time heating conditions is also likely due to differences in local load factors among these studies. In short, tar evolution in rapid heating conditions cannot be interpreted apart from a detailed consideration of the specific heat transfer characteristics of the reactor used in the investigation. Tar devolatilization properties do not, in general, exist separately in a given coal type in a precisely defineable chemical form. They are instead incompletely defined potentialitics realized in more definite form only in interaction with a specific devolatilization system, that is, heat transfer field. 
Table I

Elemental Composition of Bituminous Coals Used in Heated Grid Investigations of Tar Evolution

\begin{tabular}{lccccc} 
& \multicolumn{6}{c}{ DAF Composition } \\
Investigator(s) & $\ldots \mathrm{C}$ & $8 \mathrm{H}$ & $8 \mathrm{~N}$ & $8 \mathrm{~S}$ & 80 \\
Desypris, et.al. & 86.00 & 5.70 & 1.85 & 1.00 & 5.60 \\
Suuberg, et.al. & 76.44 & 5.41 & 1.47 & 5.97 & 9.13 \\
Unger and Suuberg & 84.28 & 5.56 & 1.68 & 1.05 & 7.02 \\
Oh & 79.37 & 5.70 & 1.35 & 5.30 & 10.23 \\
Bautista & 84.50 & 5.77 & 1.73 & 1.33 & 6.50 \\
UTRC & 84.70 & 5.40 & 1.71 & 0.92 & 7.26
\end{tabular}




\section{REFERENCES}

1. Desypris, J., Murdocil, P. and Williams, A. Fuel, 61, 807 (1982).

2. Suuberg, E.M., Peters, W.A. and Howard, J.B., Comb. Symp. (Int.), The Combustion Institute, 117 (1979).

3. Unger, P.E. and Suuberg, E.M., Fuel, 606 (1984).

4. Oh, Myongsook Susan, "Softening Coal Pyrolysis.", D.SC., MIT, Dept. of Cher. Engineering (1983).

5. Bautista, J.R., Russel, W.B. and Saville, D.A., Ind. Eng. Chem. Fundamental, 25, 536 (1986).

6. Gibbins-Matham, Jon and Kandiyoti, Rafael. Fuel Chemistry Preprints, American Chemical Society, 32, No.4, 318 (1987).

7. Gibbins-Matham, Jon. Personal Communication (1988).

8. Beuhler, R.J. and Friedman, L., Int. Jr. of Mass Spect. and Ion Processes, 78, 1 (1987).

9. Johnson, R.E., Int. Jr. of Mass Spect. and Ion Processes, 78, 357 (1987). 
Fig. I

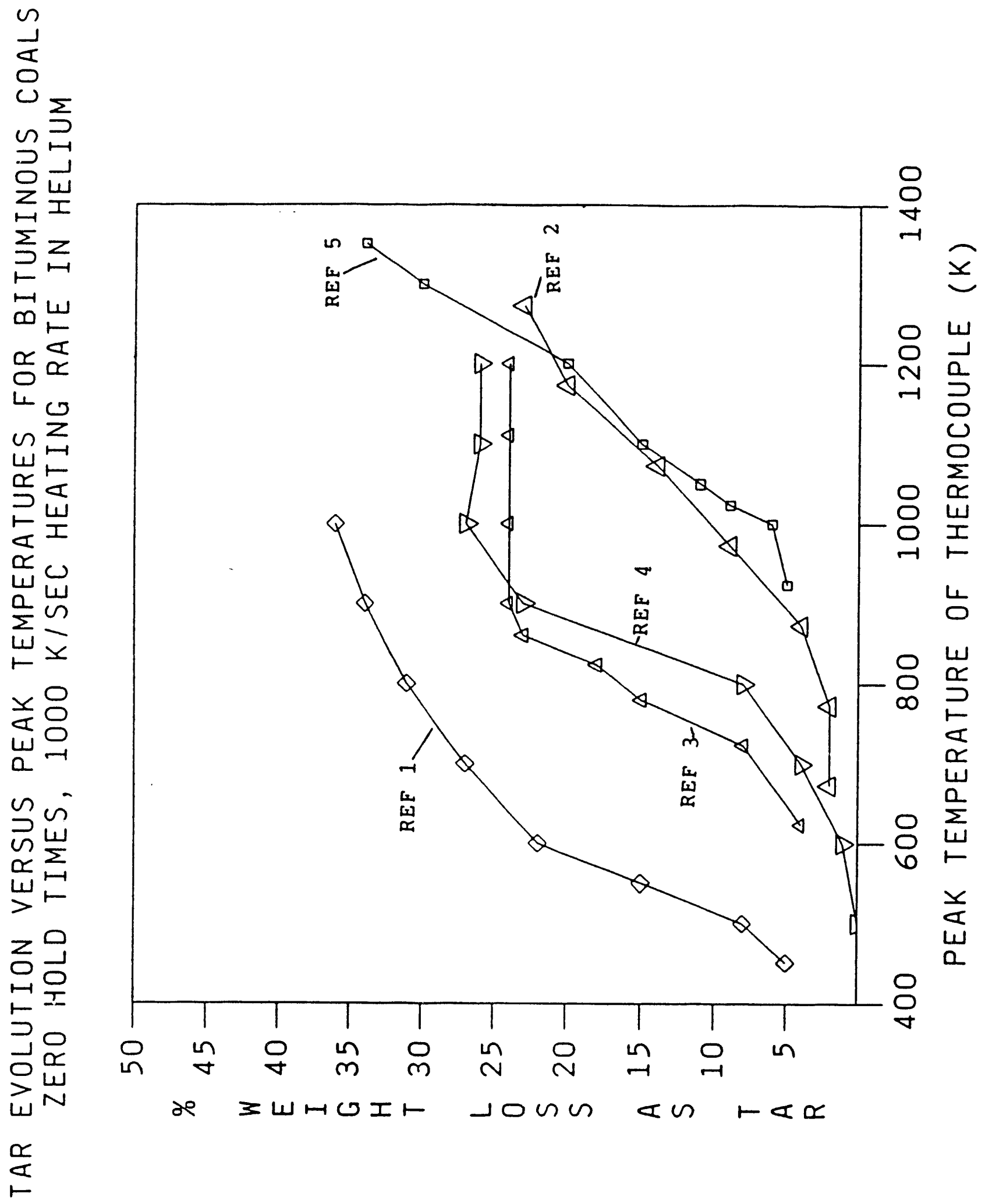


Fig. 2

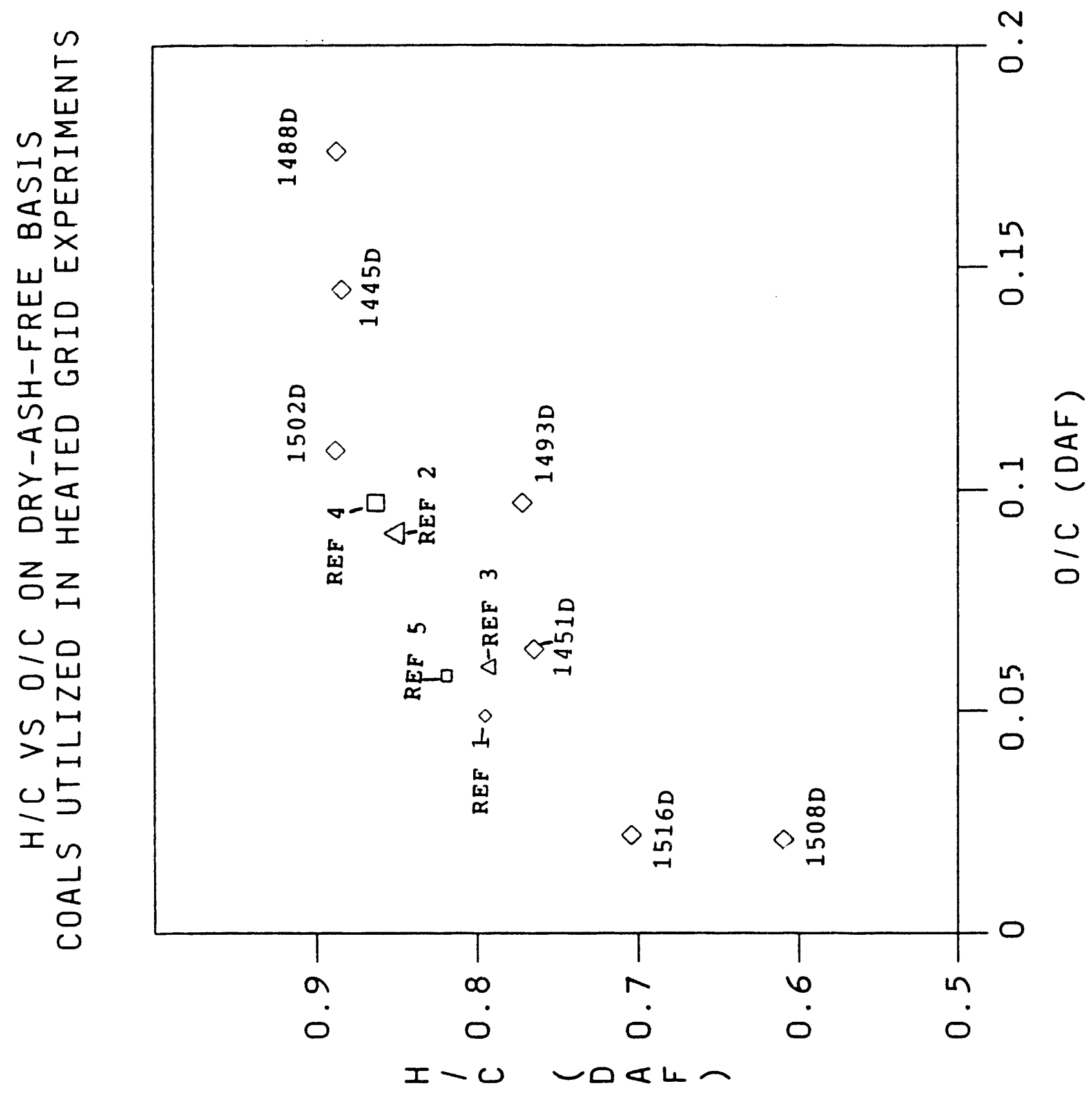


Fig. 3

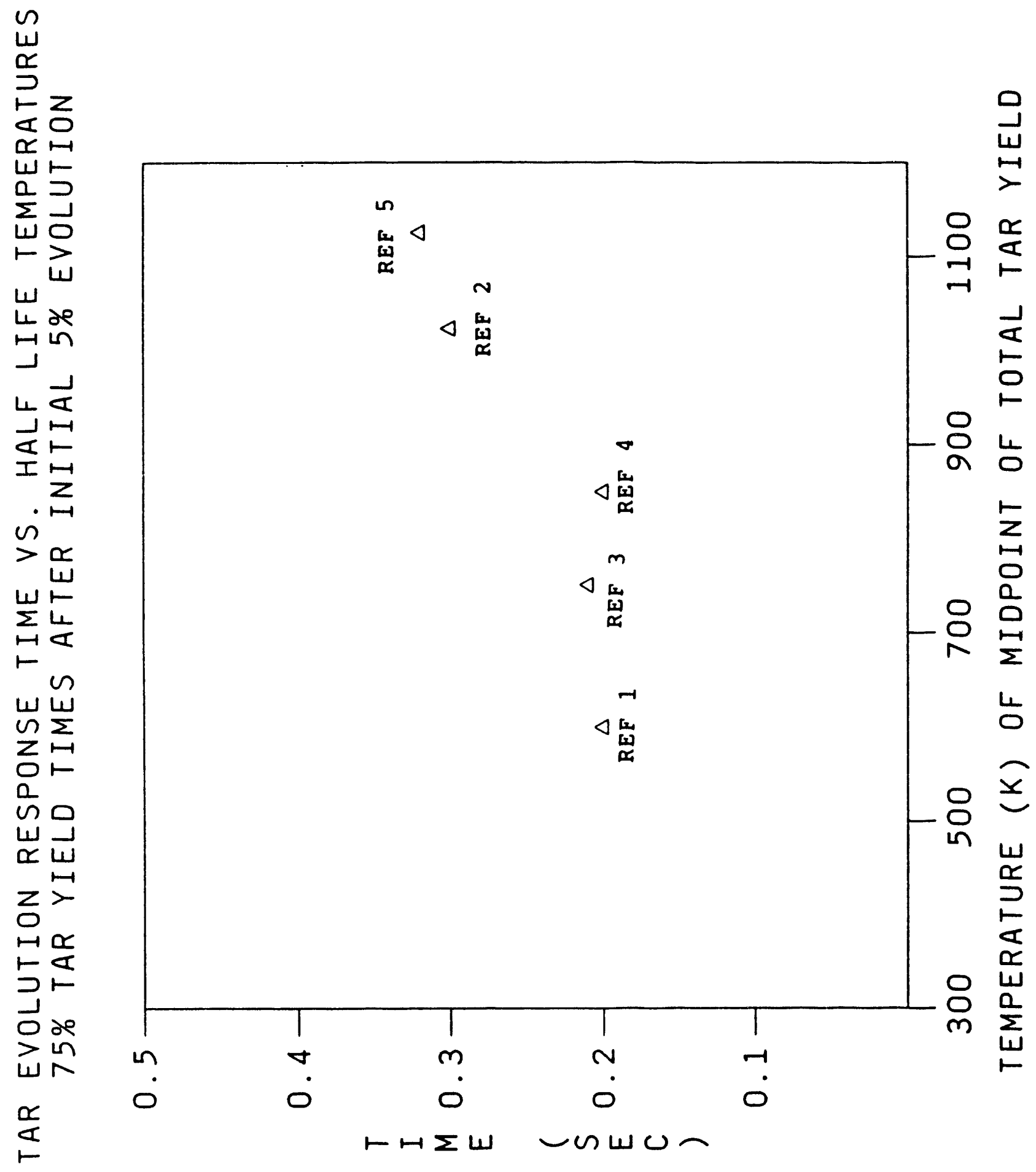


Fig. 4

$$
\frac{0}{0}
$$

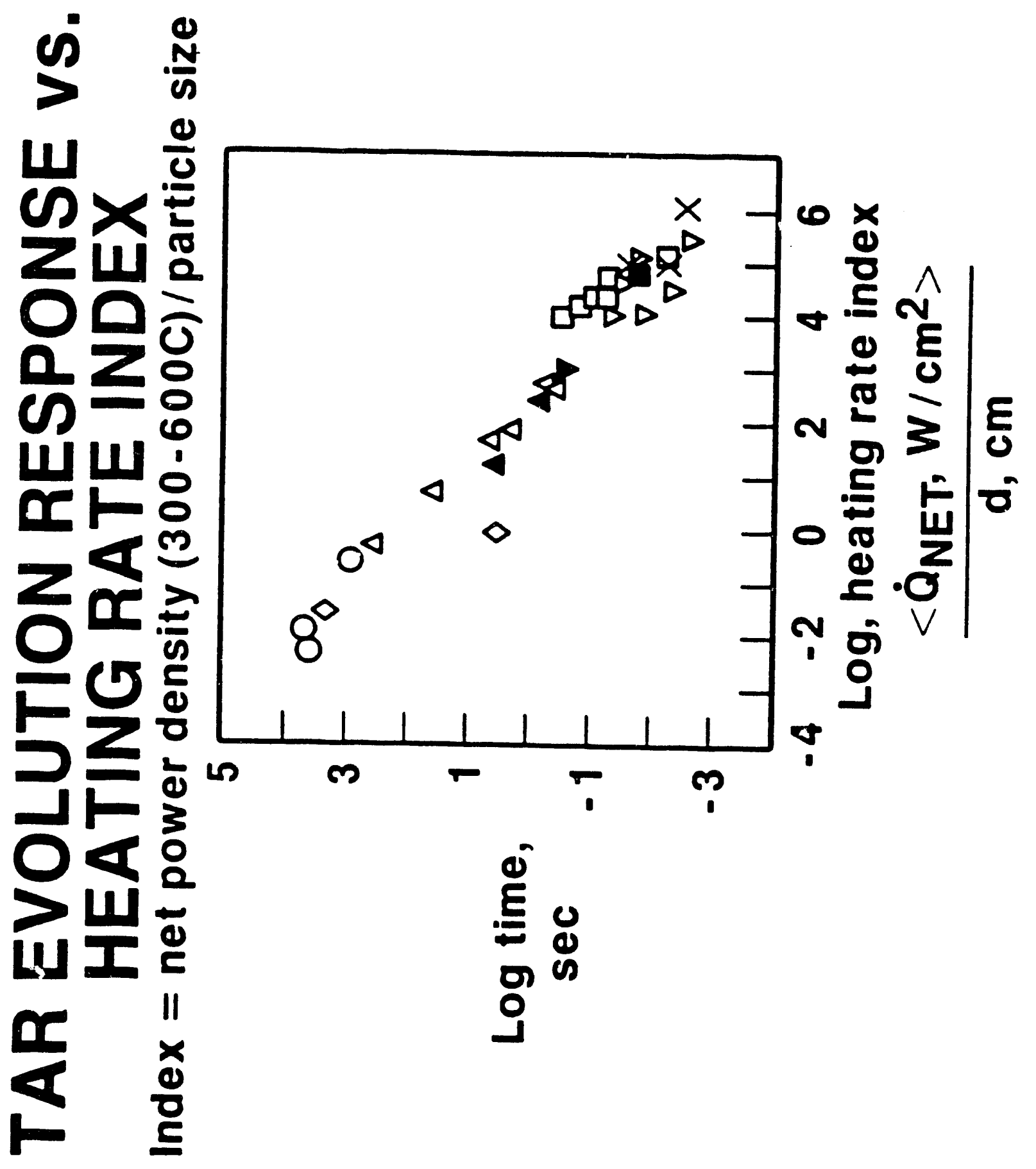


Fig. 5

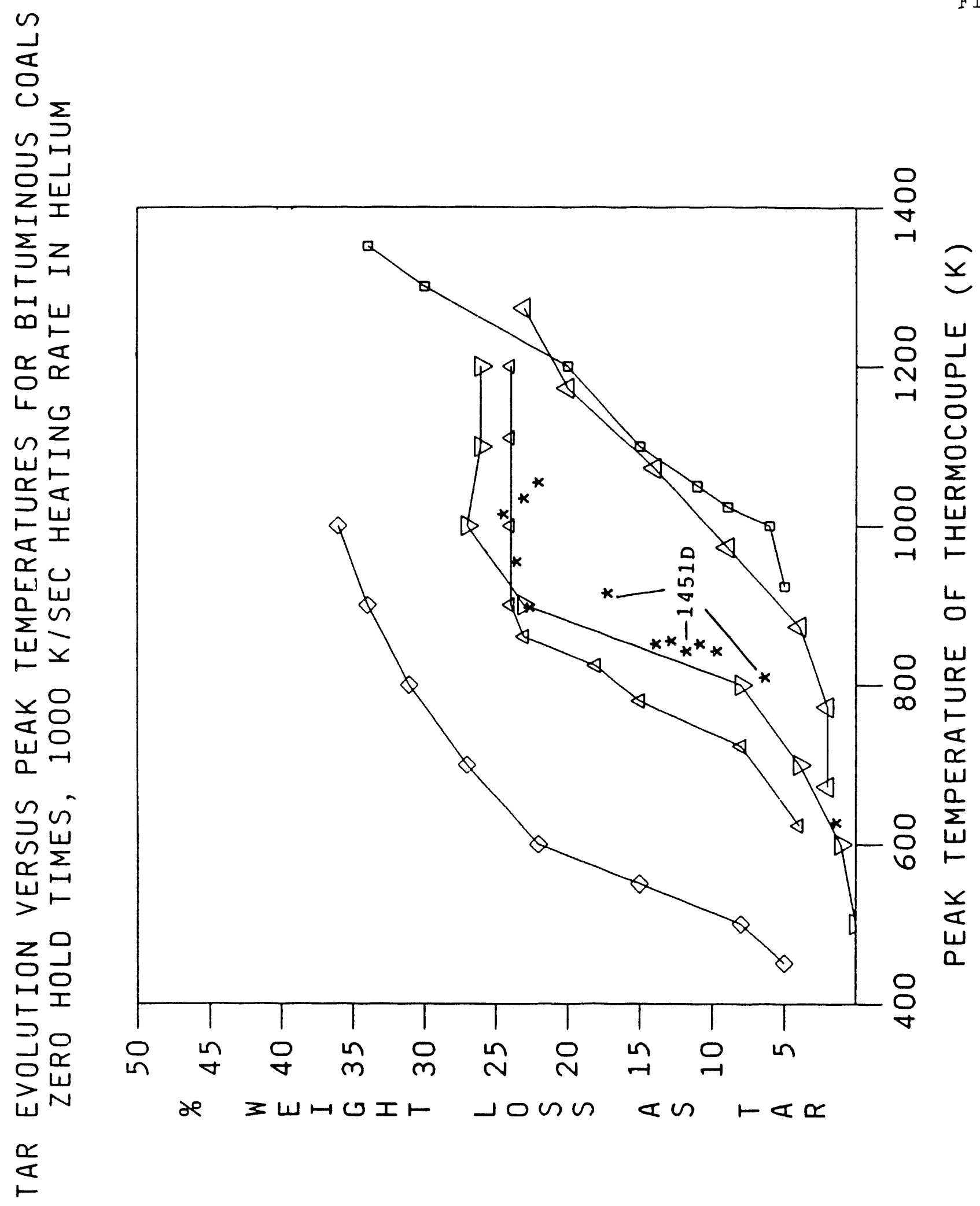




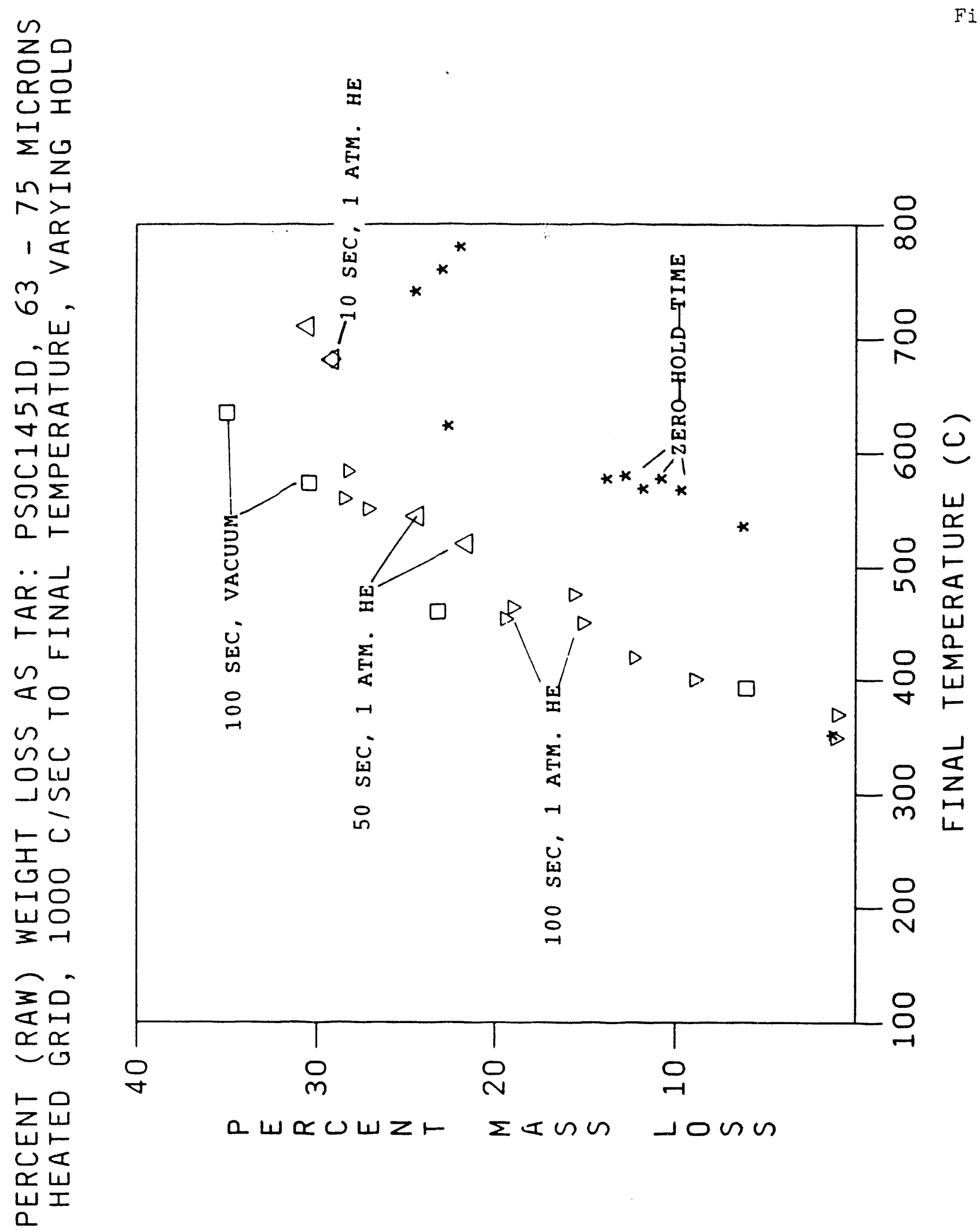


Fig. 7

番
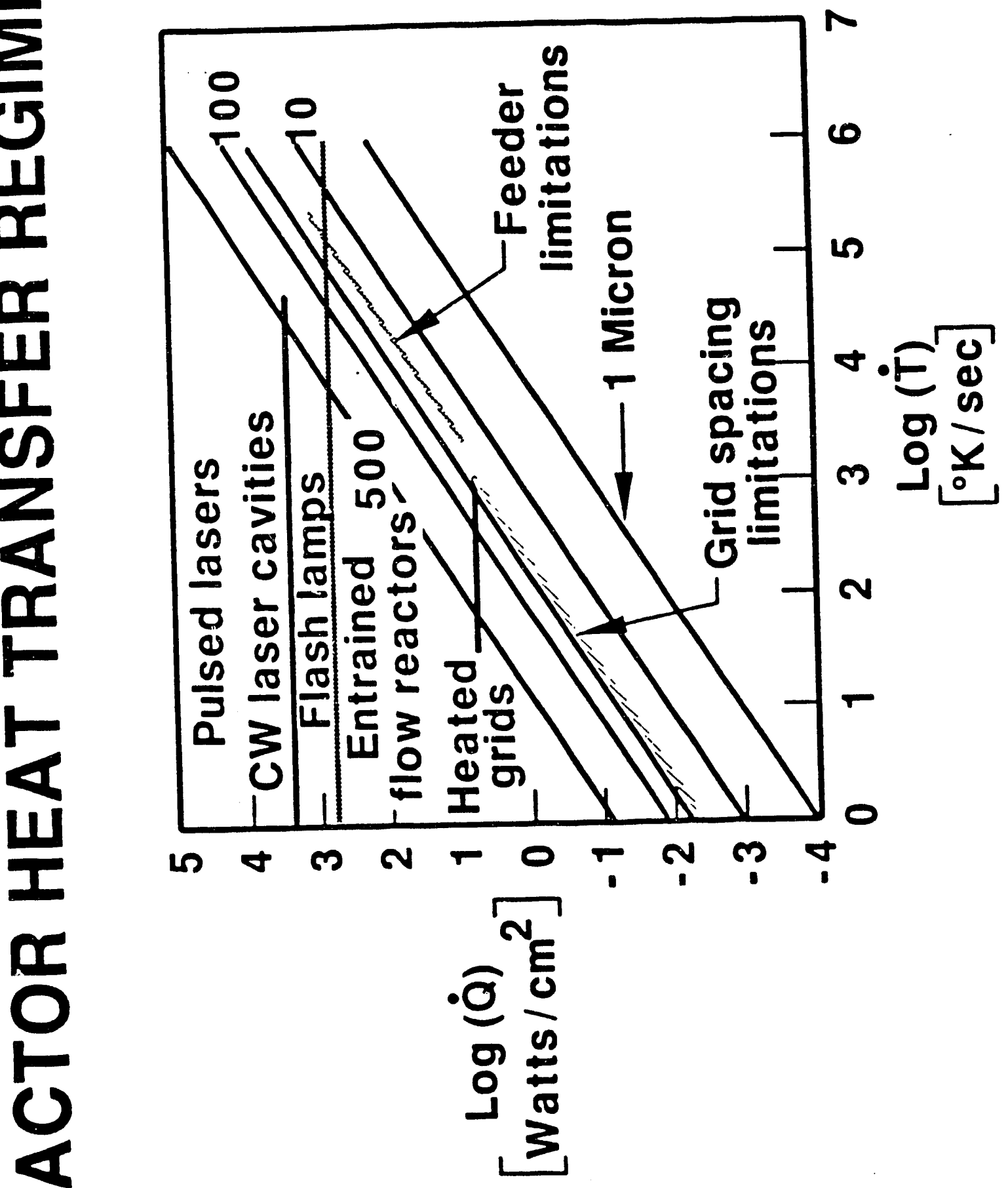

UI

$\boldsymbol{r}$ 


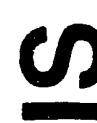

(

$\frac{1}{0}$

r

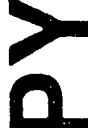

$z$

O

$F$

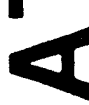

N

I

E

$F$

$\checkmark$

$-$

0

$\rightarrow$

U1

อ

$\frac{1}{0}$

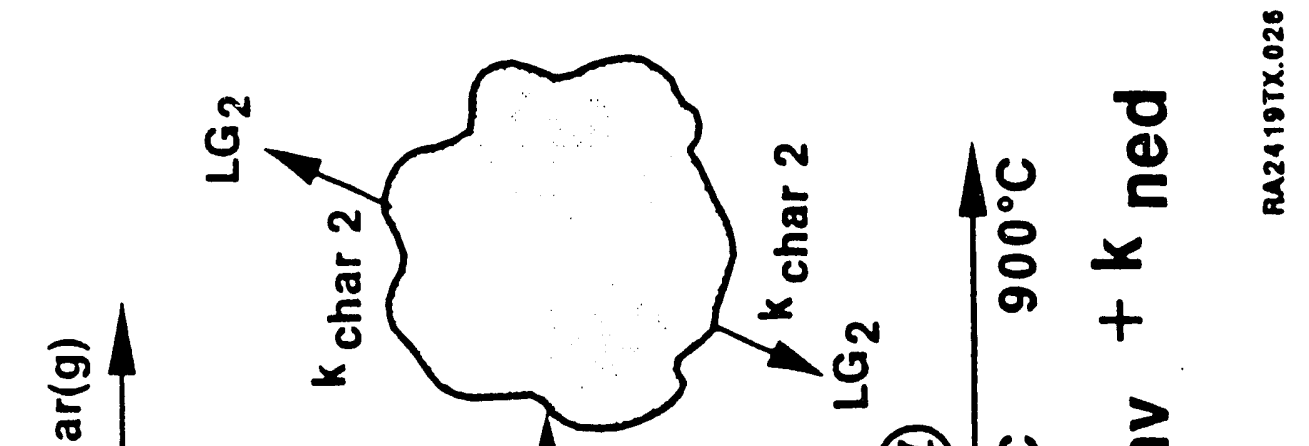

可

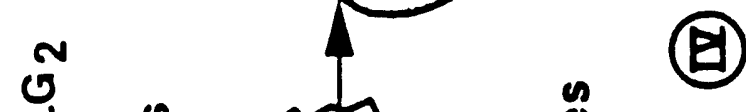

$+$

$\begin{array}{ll}0 \\ 0 \\ 0 & 0 \\ 0 & 0 \\ 0 & 0 \\ 0 & 0\end{array}$
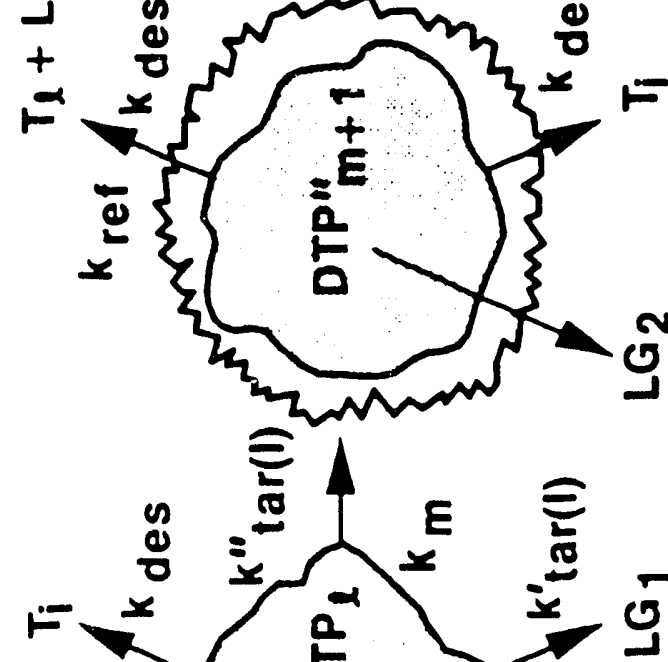

म 


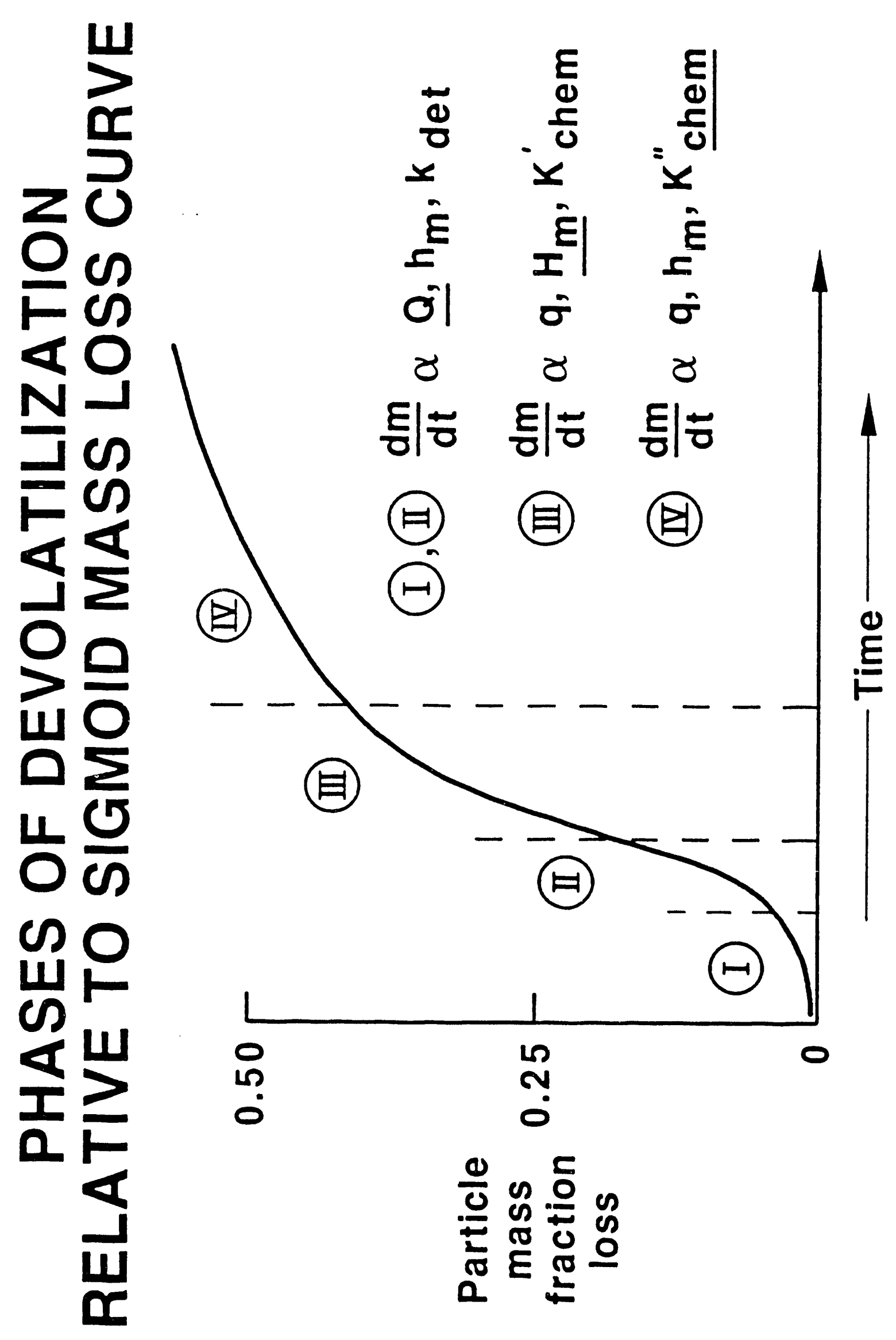



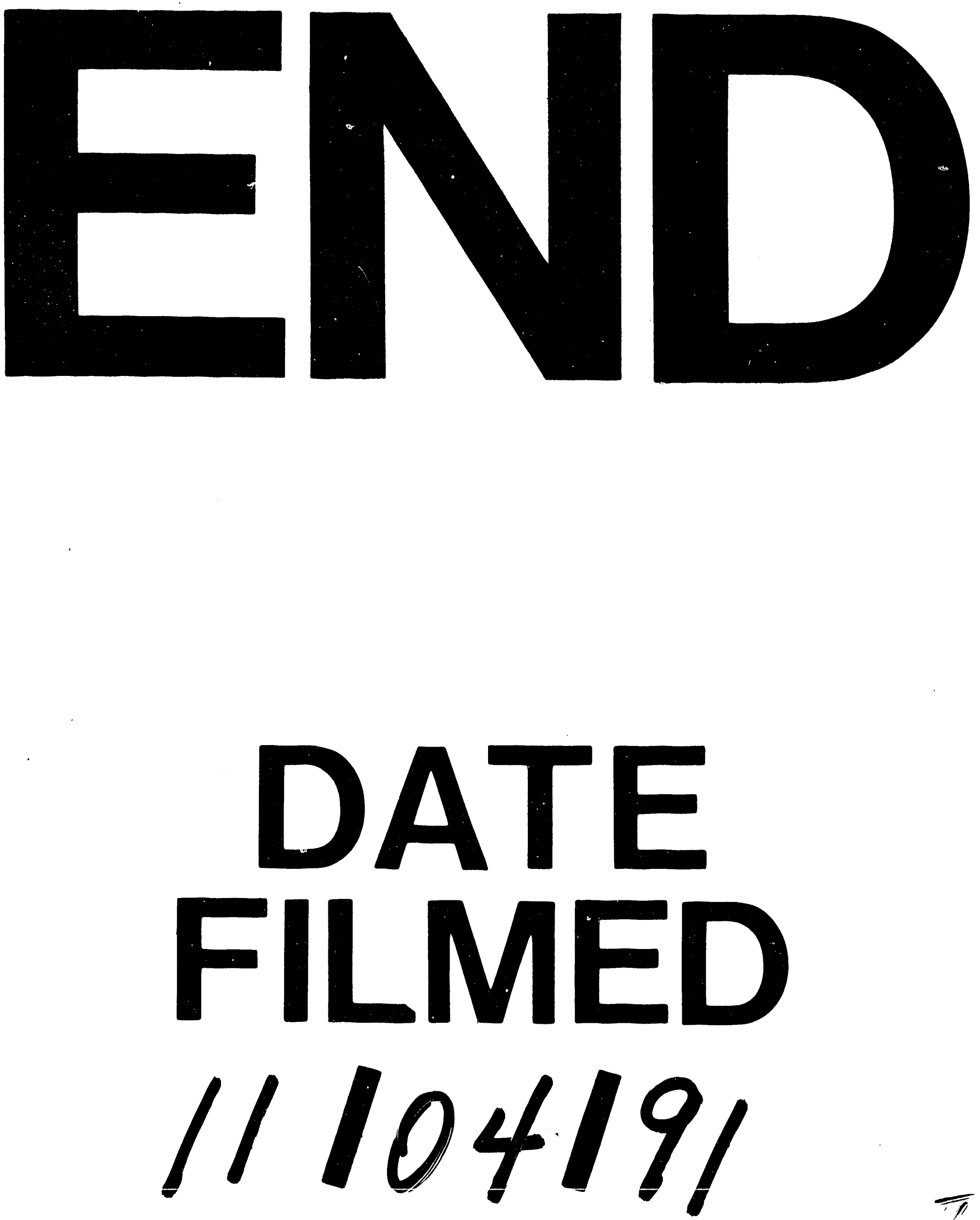

II 
